\title{
EXISTENCIA GLOBAL Y COMPORTAMIENTO ASINTÓTICO PARA UNA ECUACIÓN DE KIRCHOFF CON TÉRMINO DISIPATIVO DE COEFICIENTE VARIABLE
}

\author{
Cabanillas Lapa, Eugenio ${ }^{1}$
}

\begin{abstract}
Consideramos una ecuación de onda no lineal, con un término disipativo del tipo $a(x) u^{\prime}$ (el coeficiente a depende de la variable espacial).

Usando el método de FAEDO-GALERKIN, y definiendo funcionales, de energía adecuados, encontraremos existencia global de la solución para datos pequeños. Una vez hecho esto usamos desigualdades integrales para mostrar que la energía del sistema decae a cero de manera exponencial.
\end{abstract}

\section{INTRODUCCIÓN}

En este trabajo estudiamos la existencia global de la solución, así como el comportamiento asintótico de la energía del sistema.

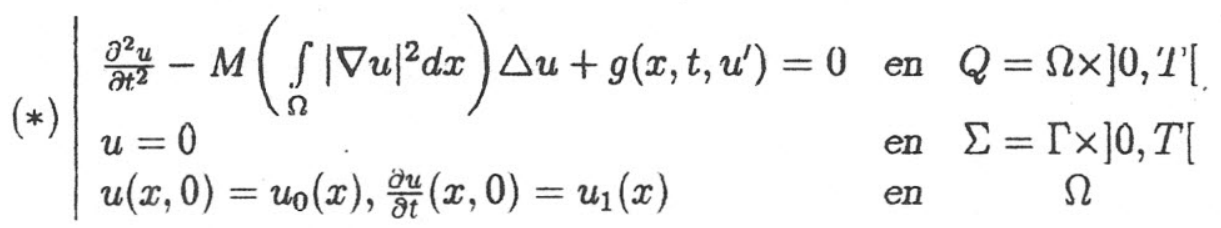

donde $\Omega$ es un abierto de $\mathbf{R}^{m}$, con frontera $\Gamma=\partial \Omega$ bien regular, $T>$ $0, M \in C^{\prime}([0,+\infty[), g$ es una función de valor real satisfaciendo condiciones apropiadas. Diversos autores: HASOYA-YAMADA [1], PATCHEU [4], HERD [2],etc., han estudiado la solubilidad global de (*) para los casos: $g\left(x, t, u^{\prime}\right)=\delta, g\left(u^{\prime}\right), C(t) u^{\prime}$. A nuestro conocimiento aún no se

\footnotetext{
${ }^{1}$ Instituto de Investigación de la Facultad de Ciencias Matemáticas - UNMSM

e-mail: d230101@unmsm.edu.pe
} 
ha abordado el estudio del importante caso $g\left(x, t, u^{\prime}\right)=a(x) u^{\prime}$, por lo que emprendimos la investigación de este problema.

\section{PRELIMINARES}

Con $(.,),. 1 . \mid$ denotamos el producto interno y la norma de $L^{2}(\Omega)$, respectivamente $H^{m}(\Omega)$ es el espacio de Sobolev usual.

Denotamos con $C_{\Omega}$ y $C_{\Omega}^{*}$ las constantes de inmersión que realizan las desigualdades:

$$
\begin{gathered}
|u| \leq C_{\Omega}|\nabla u|, \quad \forall u \in H_{0}^{1}(\Omega) \\
|\nabla u| \leq C_{\Omega}^{*}|\triangle u|, \quad \forall u \in H_{0}^{1}(\Omega) \cap H^{2}(\Omega)
\end{gathered}
$$

\section{EL RESULTADO PRINCIPAL}

Teorema 2.1.- Sea $M:[0,+\infty[\longrightarrow \mathbf{R}$ tal que:

(2.1) $M \in C^{\prime}\left(\left[0,+\infty[), M(s) \geq m_{0}>0, \forall s \geq 0\right.\right.$

(2.2) $\tilde{M}(s)=\int_{0}^{s} M(z) d z \leq s M(s)$

Sea $a \in L^{\infty}(\Omega) \cap C^{2}(\Omega)$ tal que:

(2.3) $a(x) \geq a_{0}>0, \forall x \in \Omega ;|\triangle a(x)| \leq C_{0} a(x) ;|\nabla a(x)|^{2} \leq C_{1} a(x)$ donde $C_{0}$ y $C_{1}$, son constantes positivas.

Si $u_{0} \in H_{0}^{1}(\Omega) \cap H^{2}(\Omega), u_{1} \in H_{0}^{1}(\Omega)$, entonces existe un número positivo $\varepsilon_{0}$ tal que si:

$$
\left.\left.2 C_{0} k_{1}|| \nabla u_{1}\right|^{2}+M\left(\left|\nabla u_{0}\right|^{2}\right)\left|\Delta u_{0}\right|^{2}\right]<\varepsilon_{0}
$$

$\operatorname{con} k_{1}=\operatorname{máx}\left\{\frac{3}{2 C_{0}}, C_{\Omega}+C_{\Omega}^{*}\right\}$,

entonces existe una función $u: \Omega x] 0, T[\longrightarrow \mathbf{R}$ tal que:

(2.4) $u \in L^{\infty}\left(0, T ; H_{0}^{1}(\Omega) \cap H^{2}(\Omega)\right)$

(2.5) $u^{\prime} \in L^{\infty}\left(0, T ; h_{0}^{1}(\Omega)\right)$ 
(2.6) $u^{\prime} \in L^{\infty}\left(0, T ; L^{2}(\Omega)\right)$

(2.7) $\frac{d}{d t}\left(u^{\prime}(t), w\right)+M\left(|\nabla u(t)|^{2}\right)(-\Delta u(t), w)+\left(a(x) u^{\prime}(t), w\right)=0$

$\forall w \in H_{0}^{1}(\Omega)$, en el sentido de $D^{\prime}(0, T)$.

(2.8) $u(0)=u_{0}, u^{\prime}(0)=u_{1}$

\section{Demostración:}

Usaremos el método de FAEDO-GALERKIN.

Sea $\left\{w_{1}, w_{2} \ldots, w_{j}, \ldots\right\}$ base Hilbertiana de $H_{0}^{1}(\Omega) \cap H^{2}(\Omega)$ formada por los vectores propios de $-\Delta$, esto es:

$$
-\Delta w_{j}=\lambda_{j} w_{j} ;\left.w_{j}\right|_{\Gamma}=0
$$

Consideremos $V_{m}=\left[w_{1}, w_{2}, \ldots, w_{m}\right]$ el subespacio de $H_{0}^{1}(\Omega)$ generado por $\left[w_{1}, w_{2}, \ldots, w_{m}\right]$. Buscamos una solución $u_{m}(t)$ de la forma:

$$
u_{n n}(t)=\sum_{j=1}^{m} g_{j m}(t) w_{j}
$$

donde $g_{j m}$ están determinados por el sistema:

$$
\begin{aligned}
& \left(u_{m}^{\prime \prime}(t), w\right)-M\left(\left|\nabla u_{m}(t)\right|^{2}\right)\left(\Delta u_{m}(t), w\right)+\left(a(x) u_{m}^{\prime}(t), w\right)=0, \\
& \forall w \in V_{m}
\end{aligned}
$$

(2.10) $u_{m}(0)=u_{0 m} \longrightarrow u_{0}$ en $H_{0}^{1}(\Omega) \cap H^{2}(\Omega)$

(2.11) $u_{m}^{\prime}(0)=u_{1 m} \longrightarrow u_{1}$ en $H_{0}^{1}(\Omega)$

Por el Teorema de Caratheodory existe una solución local $u_{m}(t)$ en $\left[0, T_{m}[\right.$. Las estimativas a priori permiten la solució al intervalo $[0, T]$ independiente de $m$.

\section{Estimativa a Priori I}

Haciendo $w=2 u_{m}^{\prime}(t)$ en $(2.9)$ :

$$
\left.\left(u u_{m}^{\prime \prime}(t), 2 u_{m}^{\prime}(t)\right)+M\left(\left|\nabla u_{m}(t)\right|^{2}\right)\left(\nabla u_{m}(t), 2 \nabla u_{m}^{\prime}(t)\right)+a(x) u_{m}^{\prime}(t), 2 u_{m}^{\prime}(t)\right)=0
$$


(2.12) $\frac{d}{d t}\left\{\left|u_{m}^{\prime}(t)\right|^{2}+\tilde{M}\left(\left|\nabla u_{m}(t)\right|^{2}\right)\right\}=-\int_{\Omega} 2 a(x)\left[u_{m}^{\prime}(t)\right]^{2} d x$

De (2.12) y observando que $\tilde{M}(s) \geq m_{0} s$ resulta

(2.13) $\left|u_{m}^{\prime}(t)\right|^{2}+m_{0}\left|\nabla u_{m}(t)\right|^{2} \leq E_{0}(0)=\left|u_{1}\right|^{2}+\tilde{M}\left(\left|\nabla u_{0}\right|^{2}\right)$

\section{Estimativa a Priori II}

Haciendo $w=-2 \triangle u_{m}^{\prime}(t)$ en (2.9) resulta:

$$
\begin{aligned}
& \frac{d}{d t}\left\{\left|\nabla u_{m}^{\prime}(t)\right|^{2}+M\left(\left|\nabla u_{m}(t)\right|^{2}\right)\left|\Delta u_{m}(t)\right|^{2}=-2\left|a^{1 / 2} \nabla u_{m}^{\prime}(t)\right|^{2}+\right. \\
& +\int_{\Omega} \Delta a(x)\left[u_{m}^{\prime}(l)\right]^{2} d x+2 M^{\prime}\left(\left|\nabla u_{m}(l)\right|^{2}\right)\left(\nabla u_{m}^{\prime}(l), \nabla u_{m}(l)\right)\left|\Delta u_{m}(l)\right|^{2}
\end{aligned}
$$

Tomando $w=-a(x) \Delta u_{m}(t)$ en (2.9) resulta:

$$
\begin{aligned}
& \text {.15) } \frac{d}{d t}\left\{\left(u_{m}^{\prime}(t),-a \Delta u_{m}(t)\right)+\frac{1}{2}\left|a \nabla u_{m}(t)\right|^{2}\right\}=-\left|a^{1 / 2} \nabla u_{m}^{\prime}(t)\right|^{2}+ \\
& +\left(u_{m}^{\prime}(t) \nabla a, \nabla u_{m}^{\prime}(t)\right)-M\left(\left|\nabla u_{m}(t)\right|^{2}\right)\left|a^{1 / 2} \Delta u_{m}(t)\right|^{2}-\left(u_{m}^{\prime}(t) \nabla\left(a^{2}\right), \nabla u_{m}(t)\right)
\end{aligned}
$$

\section{Definimos:}

(2.16) $F_{0}(t)=\left|u_{m}^{\prime}(t)\right|^{2}+\tilde{M}\left(\left|\nabla u_{m}(t)\right|^{2}\right)$

(2.17) $E_{1}(t)=\left|\nabla u_{m}^{\prime}(t)\right|^{2}+M\left(\left|\nabla u_{m}(t)\right|^{2}\right)\left|\triangle u_{m}(t)\right|^{2}$

(2.18) $H(t)=\left(u_{m}^{\prime}(t),-a \triangle u_{m}(t)\right)+\frac{1}{2}\left|a \nabla u_{m}(t)\right|^{2}$

Se obtiene de (2.12), (2.14), (2.15)- (2.18):

$$
\begin{aligned}
\frac{d}{d t} E_{0}(t)= & -2\left|a^{1 / 2} u_{m}^{\prime}(t)\right|^{2} \\
\frac{d}{d t} E_{1}(t)= & -2\left|a^{1 / 2} \nabla u_{m}^{\prime}(t)\right|^{2}+C_{0}\left|a^{1 / 2} u_{m}^{\prime}(t)\right|^{2}+ \\
& 2 M^{\prime}\left(\left|\nabla u_{m}(t)\right|^{2}\right)\left(\nabla u_{m}^{\prime}(t), \nabla u_{m}(t)\right)\left|\triangle u_{m}(t)\right|^{2}
\end{aligned}
$$$$
\frac{d}{d t} H(t) \leq \alpha_{0}\left|a^{1 / 2} \nabla u_{m}^{\prime}(t)\right|^{2}-\frac{a_{0}}{2} M\left(\left|\nabla u_{m}(t)\right|^{2}\right)\left|\Delta u_{m}(t)\right|^{2}
$$

$$
\text { donde: } \alpha_{0}=1+\sqrt{\frac{C_{1}}{C_{0}}} C_{\Omega}+2 C_{1}\left(\frac{|a|_{\infty} C_{\Omega} C_{\Omega \Omega}^{*}}{a_{0} m_{0}}\right)^{2}
$$


48 Existencia Global y Comportamiento Astntótico para una eCuación ...

Entonces:

$$
\begin{aligned}
& \text { (2.19) } \frac{d}{d t}\left\{E_{0}(t)+\frac{1}{C_{0}} E_{1}(t)+\varepsilon I I(t)\right\}=-\left.a^{1 / 2} u_{m}^{\prime}(t)\right|^{2}-\left(\frac{2}{C_{0}}-\varepsilon \alpha_{0}\right)\left|a^{1 / 2} \nabla u_{m}^{\prime}(t)\right|^{2} \\
& -\frac{\varepsilon a_{0}}{2} M\left(\left|\nabla u_{m}(t)\right|^{2}\right)\left|\Delta u_{m}(t)\right|^{2}+\frac{2}{C_{0}} M^{\prime}\left(\left|\nabla u_{m}(t)\right|^{2}\right)\left(\nabla u_{m}(t), \nabla u_{m}(t)\right)\left|\Delta u_{m}(t)\right|^{2}
\end{aligned}
$$

Sea

$$
S(t)=E_{0}(t)+\frac{1}{C_{0}} E_{1}(t)+\varepsilon H(t)
$$

Tomando

$$
0<\varepsilon<\min \left\{\varepsilon_{1}, \frac{1}{2 C_{0} \theta_{0}}\right\}
$$

$\operatorname{con} \varepsilon_{1}=\frac{4}{C_{0}\left(a_{0}+2 \alpha_{0}\right)}, \theta_{0}=\operatorname{máx}\left\{\frac{C_{\Omega}|a|_{\infty}}{m_{0}^{1 / 2}}, \frac{1}{4} \frac{C_{\Omega}|a|_{\infty}}{m_{0}^{1 / 2}}, \frac{1}{2} \frac{|a|_{\infty}^{2} C_{\Omega}}{m_{0}}\right\}$

resulta

(2.20) $\frac{1}{2 C_{0}} E_{1}(t) \leq S(t) \leq k_{1} E_{1}(t)$

$$
k_{1}=\operatorname{máx}\left\{\frac{3}{2 C_{0}}, C_{\Omega}+C_{\Omega}^{*}\right\}
$$

Obviamente: $\frac{1}{2 C_{0}}<k_{1}$

De (2.19)(2.20)

$\frac{d}{d t} S(t) \leq-\frac{\delta}{k_{1}} S(t)+C_{2} E_{1}^{1 / 2}(t) S(t)$

donde

$$
\begin{aligned}
& \delta=\min \left\{\frac{2}{C_{0}}-\varepsilon \alpha_{0}, \frac{\varepsilon a_{0}}{2}\right\}, C_{2}=\frac{4 M_{0} F_{0}(0)}{m_{0}^{2}} \\
& M_{0}=\max _{s \in\left[0 \cdot \frac{E_{0}(0)}{m_{0}}\right]}^{\left|M^{\prime}(s)\right|}
\end{aligned}
$$


Luego:

(2.21) $\frac{d}{d t} S(t)+\left[\frac{\delta}{k_{1}}-C_{2} E_{1}^{1 / 2}(t)\right] S(t) \leq 0$

Afirmamos que

(2.22) $\frac{\delta}{k_{1}}-C_{2} E_{1}^{1 / 2}(t)>0, \forall t \in[0,+\infty[$

Supongamos absurdamente que (2.22) no se verifica.

Por la hipótesis:

$$
E_{1}(0)<2 C_{0} k_{1} E_{1}(0)<\varepsilon_{0}=\left(\frac{\delta}{k_{1} C_{2}}\right)^{2}
$$

Por la continuidad de $E_{1}(t)$, existe $\tau>0$ tal que:

$$
\begin{gathered}
E_{1}(t)<\varepsilon_{0}, \quad \forall t \in[0, \tau] \\
E_{1}(\tau)=\varepsilon_{0}
\end{gathered}
$$

De (2.22) resulta

(2.23) $\frac{d}{d t} S(t) \leq 0, \quad \forall t \in[0, \tau[$

Pero:

(2.24) $S(\tau)-S(0)=\int_{0}^{T} S^{\prime}(\xi) d \xi$.

De $(2.20),(2.23)$ y $(2.24)$ :

$E_{1}(\tau) \leq 2 C_{0} S(\tau) \leq 2 C_{0} S(0) \leq C_{0} k_{1} E_{1}(0)<\varepsilon_{0}$

lo que es una contradicción. Así hemos mostrado que (2.22) se verifica, de donde resulta:

$$
\begin{aligned}
& \left|\nabla u_{m}^{\prime}(t)\right| \leq \text { Constante } \\
& \left|\triangle u_{m}(t)\right| \leq \text { Constante }
\end{aligned}
$$




\section{Estimativa a Priori III}

Haciendo $w=u_{m}^{\prime \prime}(t)$ en (2.9), usando las estimativas I y II, y la Inmersión de Sobolev, obtenemos de manera standard:

$$
\left|u_{m}^{\prime \prime}(t)\right| \leq \text { Constante }
$$

Las estimativas I. II y III permiten el pasaje al límite.

Teorema 2.2.- Sea $u$ la solución obtenida en el teorema 2.1. Entonces existen constantes positivas $\mu \mathrm{y} \omega$ tales que:

$$
E_{0}(t) \leq \mu e^{-\omega t}, \quad \forall t \geq 0
$$

Demostración.- Esta basada en una desigualdad integral, que aparece en KOMORNIK [3].

\section{BIBLIOGRAFIA}

[1] Hasoya, M Yameda, Y., On some nonlinear wave equations II-global existence and energy decay of solutions $J$. of the Fac. Of Sci., Univ. of Tokyo, Vol.38 $N^{\circ}$ 2, pp. 239-250(1991).

[2] Heard, M.L., A Quasilinear Hyperbolic Integrodifferential equation related to a nonlinear string. Trans. Amer. Math Soc. Vol.285 $N^{\circ} 2$ Oct. pp.805-823, (1984).

[3] Komornik, V., Decay estimates for the wave equation with internal camping Proceedings of the conference on Control Theory. Varau 1993. Birkhause Verlag. Basel, International Series Num. Analysis. 118 pp. 253-266 (1994).

[4] Patcheu, S.K., Global Existence and Exponential Decay Estimates for a Damped quasilinear Equation Comm. Part. Diff. Eq. 22 (11 and 12), pp.2007-2024. (1997). 